\title{
Outbreak of Yersinia pseudotuberculosis O:1 infection associated with raw milk consumption, Finland, spring 2014
}

T Pärn ${ }^{12}$, S Hallanvuo ${ }^{3}$, S Salmenlinna ${ }^{1}$, A Pihlajasaari ${ }^{4}$, S Heikkinen ${ }^{5}$, H Telkki-Nykänen ${ }^{6}$, M Hakkinen ${ }^{3}$, J Ollgren ${ }^{1}$, S Huusko ${ }^{1}$, R Rimhanen-Finne ${ }^{1}$

1. National Institute for Health and Welfare (THL), Department of Infectious Diseases Surveillance and Control, Helsinki, Finland

2. European Programme for Intervention Epidemiology Training (EPIET), European Centre for Disease Prevention and Control (ECDC), Stockholm, Sweden

3. Finnish Food Safety Authority Evira, Research and Laboratory Department, Food and Feed Microbiology Unit, Helsinki, Finland

4. Finnish Food Safety Authority Evira, Control Department, Food Hygiene Unit, Helsinki, Finland

5. City of Porvoo, Environmental Health Department, Porvoo, Finland

6. City of Porvoo Health Center, Finland

Correspondence: Ruska Rimhanen-Finne (Ruska.rimhanen-finne@thl.fi)

Citation style for this article:

Pärn T, Hallanvuo S, Salmenlinna S, Pihlajasaari A, Heikkinen S, Telkki-Nykänen H, Hakkinen M, Ollgren J, Huusko S, Rimhanen-Finne R. Outbreak of Yersinia pseudotuberculosis 0:1 infection associated with raw milk consumption, Finland, spring 2014. Euro Surveill. 2015;20(40):pii=30033. DOI: http://dx.doi. org/10.2807/1560-7917.ES.2015.20.40.30033

Article submitted on 31 December 2014 / accepted on 04 June 2015 / published on o8 October 2015

In March 2014, a Yersinia pseudotuberculosis (YP) outbreak was detected by a municipal authority in southern Finland. We conducted epidemiological, microbiological and traceback investigations to identify the source. We defined a case as a person with YP infection notified to the National Infectious Disease Registry between February and April 2014, or their household member, with abdominal pain and fever $\geq 38^{\circ} \mathrm{C}$ or erythema nodosum. Healthy household members were used as household-matched controls. We identified 43 cases and 50 controls. The illness was strongly associated with the consumption of raw milk from a single producer. The odds ratio of illness increased with the amount of raw milk consumed. Also previously healthy adults became infected by consuming raw milk. Identical YP strains were identified from cases' stool samples, raw milk sampled from a case's refrigerator and from the milk filter at the producer's farm. The producer fulfilled the legal requirements for raw milk production and voluntarily recalled the raw milk and stopped its production. We advised consumers to heat the raw milk to $72{ }^{\circ} \mathrm{C}$ for $15 \mathrm{~S}$. Current legislation for raw milk producers should be reviewed and public awareness of health risks linked to raw milk consumption should be increased.

\section{Background}

Yersinia pseudotuberculosis (YP) is a pathogen transmitted to humans via the faecal-oral route through consuming contaminated water or food, especially raw and undercooked products [1]. It causes yersiniosis, a gastrointestinal disease characterised by abdominal pain and fever, which can lead to post-illness complications such as erythema nodosum and reactive arthritis [2].
The incubation period of YP is three to seven days [2] but can be up to 18 days [3].

In Finland, YP infection is a notifiable disease. Outbreaks cause large variation in the annual YP incidence, for example 4.8 per 100,000 in 2006 and 0.7 per 100,000 in 2013 [4]. Most cases are sporadic, but 10 YP outbreaks were reported between 1997 and 2008 [3]. Identified sources of previous YP outbreaks include carrots [3], iceberg lettuce [5] and vegetable juice [6].

On 27 March 2014, one local public health authority in southern Finland contacted the National Institute for Health and Welfare (THL) to notify four cases of YP infection. This was followed by a notification to the National Registry for Food and Waterborne Outbreaks. We investigated the outbreak to identify its extent and source in order to apply appropriate control measures and to prevent further cases and future outbreaks.

\section{Methods}

Case finding and hypothesis generation In order to detect YP infection cases, we sent an alert to physicians and clinical laboratories all over Finland, asking them to take samples from patients with symptoms resembling yersiniosis and to report laboratoryconfirmed cases to the outbreak investigation team by phone. Clinical laboratories are required by law to notify the National Infectious Disease Registry (NIDR) of any YP findings identified in stool or of antibodies in serum. We line-listed persons with YP infection notified to the NIDR from February to April 2014. 


\section{FIGURE 1}

Number of Yersinia pseudotuberculosis cases by week of symptom onset and timeline of public health events, Finland, February-April $2014(\mathrm{n}=36)$

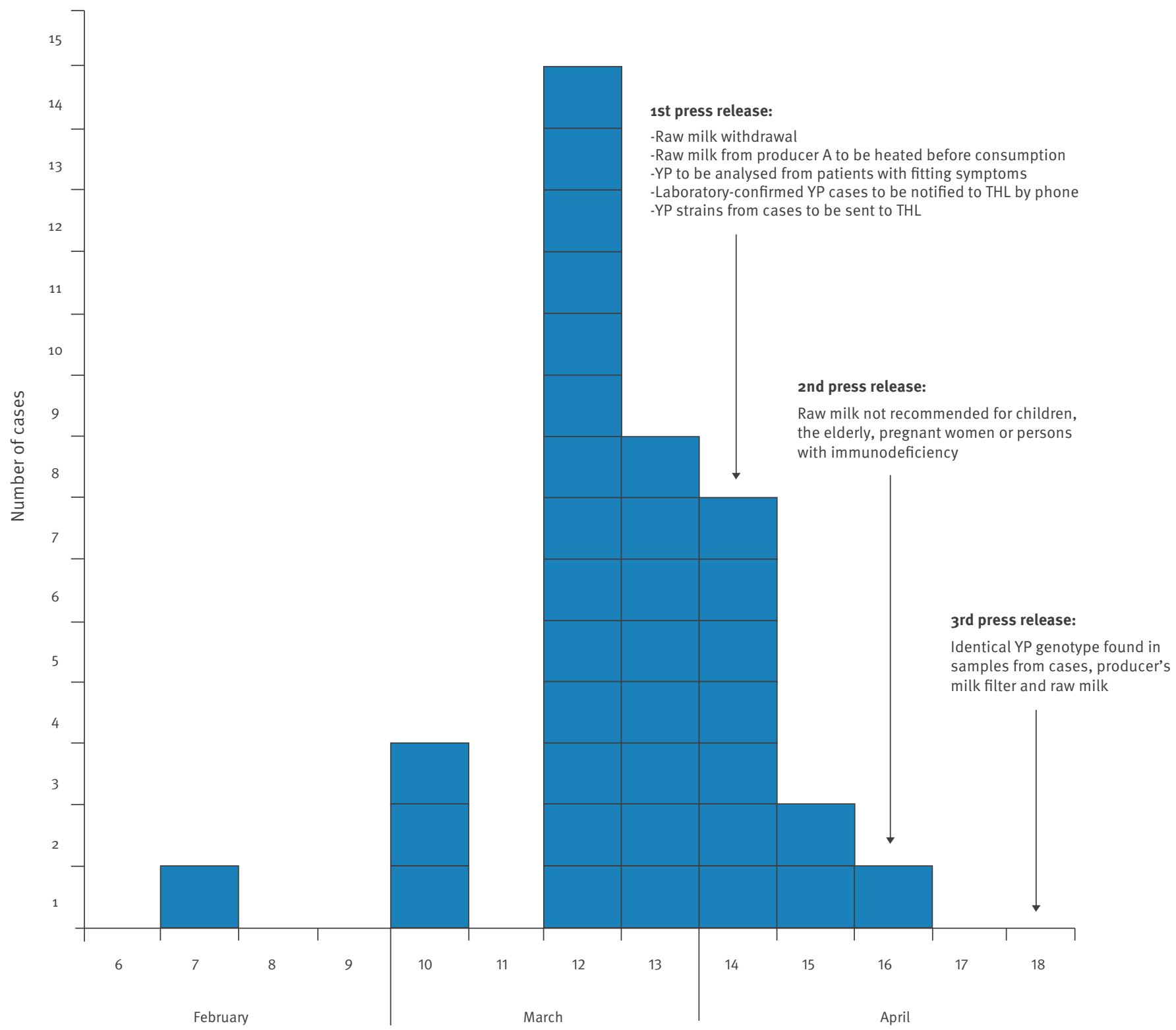

Week and month of symptom onset

THL: National Institute for Health and Welfare; YP: Yersinia pseudotuberculosis.

Date of symptom onset available for 36 cases.

A public health nurse interviewed six persons from the line list, using a standard trawling questionnaire to generate hypotheses. The trawling questionnaire for gastrointestinal illnesses contained 97 questions on the clinical picture of the disease, travel history, consumed foods and drinks, cooking methods, sites for shopping and eating out, and animal contact.

\section{Case-control study and statistical analysis}

We conducted a case-control study to test the hypothesis suggested by the trawling interviews. Our study population comprised persons with YP infection notified to the NIDR and their household members. The outbreak team at THL developed a web-based questionnaire. This included questions about the demographical and clinical characteristics of the subjects, onset of the symptoms, food exposure suggested by trawling interviews and by literature as previously identified sources of YP outbreaks, and about the frequency and amount of foods consumed during the two weeks before the onset of symptoms. Respondents 
TABLE 1

Exposure to food items among cases and controls (matched univariate analysis), Yersinia pseudotuberculosis outbreak, Finland, February-April $2014(\mathrm{n}=93)$

\begin{tabular}{|c|c|c|c|c|}
\hline & Cases $(n=43)$ & Controls $(n=50)$ & & \\
\hline Variable & $\begin{array}{c}\text { Exposed } \\
n(\%)\end{array}$ & $\begin{array}{c}\text { Exposed } \\
\text { n (\%) }\end{array}$ & $\operatorname{mOR}(95 \% \mathrm{Cl})$ & $p$ value \\
\hline Raw milk (producer $\mathrm{A}$ ) & $42(98)$ & $24(48)$ & $22.2^{\mathrm{a}}(3.6-\infty)$ & $<0.001$ \\
\hline Raw milk (in general) & $42(98)$ & $30(60)$ & $16.9^{\text {a }}(2.6-\infty)$ & 0.001 \\
\hline Salad & $34(79)$ & $40(80)$ & $1.7(0.1-117.8)$ & 1.000 \\
\hline Raw milk (producer other than A) & $1(2)$ & $2(4)$ & $1.5^{\mathrm{a}}(0.0-58.5)$ & 1.000 \\
\hline Other vegetable & $26(60)$ & $38(76)$ & $0.9^{\mathrm{a}}(0.1-\infty)$ & 1.000 \\
\hline Carrot & $38(88)$ & $41(82)$ & $0.8(0.0-49.1)$ & 1.000 \\
\hline Vegetable juice & o (o) & $4(8)$ & $0.56^{\mathrm{a}}(0.0-7.5)$ & 0.667 \\
\hline
\end{tabular}

Cl: confidence interval; mOR: matched odds ratio.

a Median unbiased estimate.

\section{TABLE 2}

Unadjusted and adjusted dose-response relationship between consumed raw milk from producer A and Yersinia pseudotuberculosis infection, Finland, February-April $2014(\mathrm{n}=93)$

\begin{tabular}{|c|c|c|c|c|c|c|c|}
\hline & $\begin{array}{l}\text { Cases } \\
(n=43)\end{array}$ & $\begin{array}{c}\text { Controls } \\
(\mathrm{n}=50)\end{array}$ & $\begin{array}{c}\text { Total } \\
(n=93)\end{array}$ & \multicolumn{2}{|c|}{ Univariate } & \multicolumn{2}{|c|}{ Multivariable $^{a}$} \\
\hline & n (\%) & n (\%) & n (\%) & $\operatorname{mOR}(95 \% \mathrm{Cl})^{\mathrm{b}}$ & $p$ value & $\operatorname{mOR}(95 \% \mathrm{Cl})^{\mathrm{b}}$ & $\mathrm{p}$ value \\
\hline \multicolumn{8}{|c|}{ Quantity of consumed milk } \\
\hline None & $2(8)$ & $24(92)$ & $26(100)$ & \multicolumn{2}{|c|}{ Reference } & \multicolumn{2}{|c|}{ Reference } \\
\hline$<1 \mathrm{dL} /$ day & $9(56)$ & $7(44)$ & $16(100)$ & $7.2(0.71-\infty)$ & 0.098 & $10.7(0.99-\infty)$ & 0.051 \\
\hline $1-3 \mathrm{dL} / \mathrm{day}$ & $19(63)$ & $11(37)$ & $30(100)$ & $14.5(2.21-\infty)$ & 0.003 & $8.0(1.09-\infty)$ & 0.039 \\
\hline$>3 \mathrm{dL} / \mathrm{day}$ & $12(71)$ & $5(29)$ & $17(100)$ & $20.0(2.07-\infty)$ & 0.008 & $12.2(1.26-\infty)$ & 0.029 \\
\hline \multicolumn{6}{|l|}{ Age } & $0.92(0.85-0.97)$ & $<0.001$ \\
\hline
\end{tabular}

$\mathrm{Cl}$ : confidence interval; mOR: matched OR.

${ }^{a}$ Adjusted for age.

${ }^{b}$ Median unbiased estimator of OR; univariate $\mathrm{p}$ value for trend is $<0.001$; multivariable $\mathrm{p}$ value for trend is 0.016 .

\section{TABLE 3}

Environmental samples tested during a Yersinia pseudotuberculosis outbreak, Finland, February-April 2014 (n = 21)

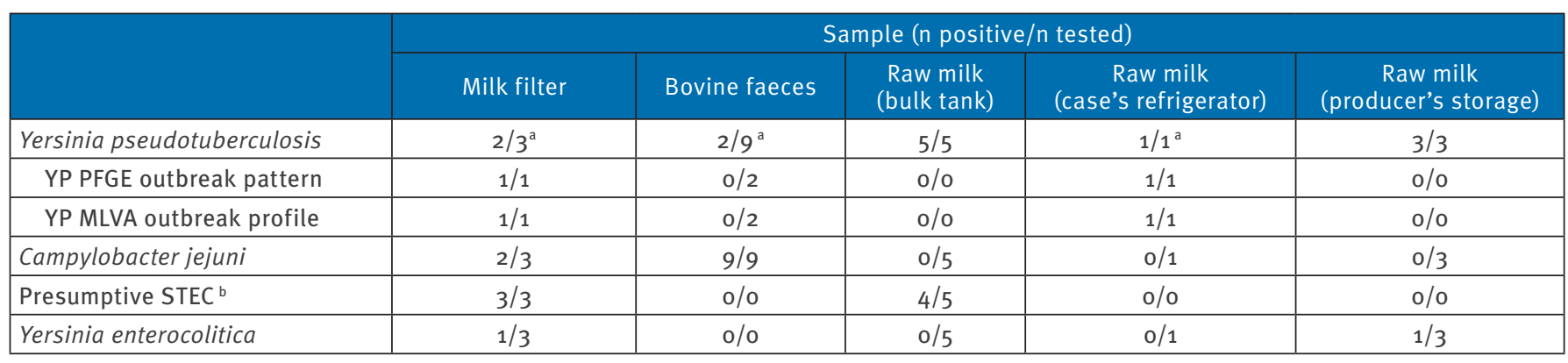

MLVA: multilocus variable-number of tandem repeats analysis; PFGE: pulsed-field gel electrophoresis; STEC: Shiga toxin-producing Escherichia coli. YP: Yersinia pseudotuberculosis.

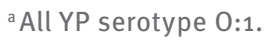

${ }^{b}$ Presumptive STEC is defined as real-time PCR detection of (i) the stx gene, (ii) stx and eae genes or (iii) stx and eae genes as well as genes associated with one of the serogroups $0157,0111,026,0103$ or 0145. 
were invited to reply using either a web-based questionnaire or a paper form.

We defined a case as a person with YP infection notified to the NIDR or with symptoms of abdominal pain and fever of $\geq 38^{\circ} \mathrm{C}$ or erythema nodosum in the period from February to April 2014. The matched controls were respondents living in the same household as the case who did not meet the case definition.

We calculated matched odds ratios to explore the associations between a single exposure and the outcome. To control for possible confounders, significant variables in the univariate analysis were included in the multivariable model. Households with only one member or no controls were excluded from the matched analysis. We coded the responses 'do not remember' or 'do not know' as missing values. We used exact conditional logistic regression to calculate odds ratios (OR) and $95 \%$ confidence intervals $(\mathrm{Cl})$ for both univariate and multivariable analyses. Where a finite conditional maximum likelihood estimate did not exist, the median unbiased estimator (MUE) of the OR was calculated [7].

To calculate the point estimates for the effects of exposure doses in the univariate analysis, we used binary variables for each milk dose in the model. In the multivariable analysis, we adjusted the model for continuous age. The significance of the dose-response trend was assessed using the ordinal level dose-response variable as continuous both in the univariate and in the multivariable analysis. For all analyses, a $p$ value of<0.05 was considered significant. The STATA Data Analysis and Statistical Software version 12 (Stata Corporation, College Station, United States) was used to perform the analysis.

\section{Traceback and microbiological investigation}

After raw milk was identified as a possible source of the outbreak, the municipal authority contacted the producer in order to investigate the production procedure, to identify when, where and how much of the specific product was distributed and to find out whether the producer followed the relevant regulations for food production.

On 4 April, an opened $3 \mathrm{~L}$ package of raw milk from a specific producer (with 'use by' date on 1 April) was sampled from a case's refrigerator. On 7 April, the local team visited the producer's farm to collect the milk filter, bulk tank milk and bovine faecal samples. Bovine faecal samples were pooled to represent ca 10 animals each. Unopened $3 \mathrm{~L}$ packages from the producer's storage were also collected.

Stool specimens from the patients were analysed at four clinical microbiology laboratories using faecal culture and a quantitative PCR for Salmonella, Yersinia, Campylobacter, Vibrio cholera, Shigella, and Shigatoxin-producing Escherichia coli (STEC) [8]. Milk, milk filter and bovine faecal samples were analysed at the Finnish Food Safety Authority Evira. Screening of environmental samples for YP, pathogenic Yersinia enterocolitica and STEC was conducted by real-time PCR [9-11]. For Y. enterocolitica detection, we followed the technical specification ISO/WD 10273 [12] and for STEC detection we followed ISO/TS 13136 [11], where presumptive STEC is defined as real-time PCR detection of (i) the stx gene, (ii) stx and eae genes or (iii) stx and eae genes as well as genes associated with one of the serogroups $\mathrm{O}_{157,} \mathrm{O}_{111}, \mathrm{O}_{26} \mathrm{O} \mathrm{O}_{103}$ or $\mathrm{O}_{145}$.

For isolation of YP, samples of $10 \mathrm{~g}$ (faeces) $25 \mathrm{~mL}$ (milk) or one half of a milk filter were homogenised in phosphate-mannitol-peptone (PMP) broth [13] as described previously [3]. The growth characteristics of YP in bulk tank milk were studied by plating dilutions of the refrigerated tank milk on CIN agar on day 0, 1, 2, 5, 7, 9 and 12 after collection (up to one day after milking). Each sample was plated in duplicate and the average count of YP colonies was used. YP isolates were identified by API 20E (bioMérieux, France) and MALDI-Biotyper (Bruker Daltonics, GmbH). Isolation of Campylobacter spp. from milk, milk filter and bovine faecal samples was carried out according to ISO 10272-1:2006 [14] with a modification in the enrichment: an incubation time of 24 hours without a pre-incubation step was used.

Serotyping, pulsed-field gel electrophoresis (PFGE) and multilocus variable-number tandem repeat analysis (MLVA) were performed at THL to characterise and compare the isolates $[5,15]$. No band differences were allowed in PFGE and a single-locus difference was allowed in MLVA to designate two strains as indistinguishable. C. jejuni isolates were subtyped at Evira with PFGE using Smal and Kpnl [16].

\section{Results}

Descriptive and analytical epidemiology

From February to April 2014, we identified and linelisted 55 persons with YP infection registered in the NIDR. They represented 48 households and their median age was 14 years (range: $1-67$ ). Of those 55, 35 were men and 51 were from the Helsinki and Uusimaa hospital district in the south of Finland. In those three months, the incidence rate of YP infection cases in this hospital district was more than six times higher (incidence rate ratio (IRR): $6.4 ; 95 \% \mathrm{Cl}: 3.0-15.6)$ than in the same period in 2013 (13.2/100,000 vs 2.1/100,000). All six persons completing the trawling interview reported consuming raw milk from a single producer $A$.

In total, 93 persons from 30 households (household response rate: 63\%) completed the questionnaire. The online questionnaire was used by 53 of 93 . Of the respondents, 43 were identified as cases (33 based on NIDR notification, 10 based on symptoms) and 50 as controls. Respondents under the median age of 23 years were 12 times more likely to be a case than those over the age of $23(\mathrm{OR}=12.1 ; 95 \% \mathrm{Cl}: 2.8-111.0$; 
$\mathrm{p}$ <0.001). Of the 43 cases, 22 were men, and the median age was 13 years (range: $1-67)$. One case had a campylobacter co-infection.

The first case fell ill on 10 February, and the number of cases peaked in March, week 12 (Figure). The median duration of the illness was 14 days (range: 4-27). Abdominal pain and fever were the most commonly reported symptoms (39 and 31 of the 43 cases, respectively), followed by nausea, diarrhoea and joint pain (20, 19 and 13 of the 43 cases, respectively). Three cases developed erythema nodosum, and four were hospitalised. No significant difference $(p=1.000)$ was found between cases and controls regarding previously diagnosed chronic diseases (7/43 vs 9/50, respectively).

Eight households, comprising in total 13 cases, were excluded from the matched analysis because they had only one household member or no controls. In the univariate analysis, raw milk was the only food item significantly associated with the illness (Table 1) and an increase of one year in age decreased the odds of illness by $6 \%(\mathrm{OR}=0.94 ; 95 \% \mathrm{Cl}$ : $0.90-0.98$; $\mathrm{p}$ <0.001). Compared with those who did not drink raw milk from producer $A$, the age-adjusted odds of illness were higher than 35 for those who consumed it (MUE of OR=35.2; $95 \% \mathrm{Cl}: 4.07-\infty ; p<0.001$ ). Of the 93 questionnaire respondents, 89 provided information on the volume of consumed raw milk. The OR of illness increased with the volume of raw milk consumed $(p=0.016)($ Table 2$)$.

\section{Traceback and microbiological investigation}

The raw milk was produced on a farm that had 90 milking cows and two regular daily employees. The majority of the milk produced was delivered to a dairy for pasteurisation. In February 2014, the farm started operating a packaging facility for the raw milk once per week. The product was first distributed to retail stores on 10 February. From 10 February to 3 April 2014, 11,835 L of this raw milk were delivered to 24 shops in southern Finland. The raw milk was sold in $3 \mathrm{~L}$ packages, with a 'use by' date five days from the date of packing. In compliance with Finnish regulations, all packages had a warning label, stating that the milk may contain harmful microbes and should be heat-treated before serving to risk groups, i.e. children, pregnant women, the elderly or individuals with a severe primary disease [17].

In the microbiological investigation, all of the $41 \mathrm{YP}$ patient isolates that had been sent to the THL reference laboratory from February to April had the serotype 0:1. Isolates from eight cases were characterised by PFGE and all showed an identical profile (outbreak profile). Seven of eight isolates were also analysed with MLVA and had the indistinguishable outbreak profile 5-9-3-2-4-8-12. YP with the outbreak profile was also detected in a milk sample from the refrigerator of a case and from the milk filter (Table 3). The concentration of YP in tank milk increased steadily during storage at $4^{\circ} \mathrm{C}$. It was 2 colony-forming units (cfu)/ $\mathrm{mL}$ when collected from the tank and $5 \mathrm{cfu} / \mathrm{mL}, 26 \mathrm{cfu} /$ $\mathrm{mL}$ and $120 \mathrm{cfu} / \mathrm{mL}$ after one, two and five days of storage, respectively. After 12 days of storage, the concentration was $3,500 \mathrm{cfu} / \mathrm{mL}$.

C. jejuni was detected by culture in environmental samples, and pathogenic $Y$. enterocolitica, presumptive STEC 0103, 0145 and STEC 0103 were detected by PCR (Table 3). C. jejuni was not isolated from tank milk. Attempts at isolation did not yield any $Y$. enterocolitica or STEC isolates. A C. jejuni isolate from one patient was available for comparison with isolates from the milk filters $(n=2)$ and bovine faeces $(n=7)$. Identical PFGE profiles were identified.

\section{Public health measures}

After producer A's raw milk was identified as the suspected source of the outbreak, the producer voluntarily recalled a total of $200 \mathrm{~L}$ of raw milk from the market on 3 April 2014. The farm discontinued the commercial production of raw milk. The local authority, THL and Evira announced the outbreak in the media in order to inform the public about the possible contamination of the raw milk from a particular producer and to advise to heat it to $75^{\circ} \mathrm{C}$ for $15 \mathrm{~s}$ before consumption (Figure).

\section{Discussion}

We describe the outbreak of 55 cases of YP infection that occurred between February and April 2014 in southern Finland. The YP infection was associated with the consumption of raw milk from a single producer, with $98 \%$ of the cases consuming raw milk from this source. Microbiological evidence supported the epidemiological findings. An identical YP serotype and genotype were found in isolates from the patients, the milk filter from producer's farm and raw milk from a case's fridge.

Critical selection of controls is necessary to avoid introducing bias in the study. We used a method in which any healthy responding household member was suitable for being a matched control for a case. Choosing controls among household members may result in overmatching [18]. Despite the fact that household members may share similar dietary habits, we were able to demonstrate a strong association between raw milk consumption and illness.

An increase in outbreaks related to raw milk contaminated by a variety of pathogens has been observed [19]. Pasteurisation is a well-known and effective way to eliminate pathogens and bacteria from raw milk, decreasing the risk of disease transmission [20]. THL and the Finnish Food Safety Authority Evira recommend that children, the elderly, pregnant women and persons with a severe primary disease should heattreat raw milk before consumption. In this study, age was associated with illness: those under 23 years of age were more likely to get ill. By contrast, previously 
diagnosed chronic diseases did not affect the probability of YP infection. Our results are in line with findings from a study showing that healthy adults can become infected by consuming contaminated raw milk [21], and we suggest that also adults should heat raw milk before consuming it.

This outbreak was identified because a cluster of YP cases visited the same hospital. In Finland, Yersinia and Campylobacter infection notifications are not monitored in real time, and strains isolated from these cases do not need to be sent to the national reference laboratory for typing. Therefore, detection of geographically widespread Yersinia or Campylobacter outbreaks and routine comparison of YP isolates would be impossible. No YP profile identical to the current outbreak strain was identified among 61 Finnish YP strains tested in a previous study [15]. In this outbreak, identification of a cluster of cases allowed a timely response and prompt control measures. The producer voluntarily discontinued raw milk production and withdrew the product from the market immediately after the trawling interviews were completed. Simultaneously, the national authorities informed the public and healthcare professionals about health risks linked to raw milk consumption. No new YP cases were detected after the recall of the raw milk.

Despite the early detection and rapid control measures, this outbreak was larger than previously reported outbreaks linked to raw milk consumption [22]. Moreover, there were probably more cases than recorded. Additional cases on the basis of symptoms were detected by us in one in four of the responding households. Persons with severe symptoms are more likely to seek medical care and thus more likely to be sampled. It has been estimated that only ca $20 \%$ of persons contracting gastroenteritis consult a physician [23].

In this outbreak, the milk was contaminated by several organisms. The most probable route for contamination was through bovine faeces during milking. YP with an identical profile was detected in patients and bovine faeces. In addition, an identical C. jejuni profile was identified in one patient, bovine faeces and the milk filter. Raw milk may also become contaminated via cleaning water, cow mastitis or cross-contamination through humans or rodents [24]. Rodents and other small mammals are the main reservoirs for YP [2]. In previous YP outbreaks in southern Finland, rodents on farms were suggested as a possible source of YP transmission $[3,25]$. Further studies are needed to clarify the transmission dynamics of YP on farms.

In this study, the case from whose refrigerator raw milk had been sampled reported consumption of less than $1 \mathrm{dL}$ of milk three days after the packaging date, and the amount of YP in tank milk was estimated at 26-120 $\mathrm{cfu} / \mathrm{mL}$ at the same time point. While the infectious dose of YP is believed to be $10^{8}$ or more bacteria if ingested orally [26], ingestion of fewer than $10^{4} \mathrm{cfu}$ of YP could have sufficed for infection in this outbreak. YP may reach infectious concentrations in products stored for at least several days at refrigerator temperature, since it can multiply at $4^{\circ} \mathrm{C}[27]$.

YP is one of the pathogens included in the European Food Safety Authority's shortlist of microbiological hazards, indicating that it can be transmitted to humans through the milk [28]. It is, however, seldom studied and little information is available about its prevalence in unpasteurised milk $[18,29,30]$. No YP was found in raw cow milk samples from bulk tanks collected from 183 dairy farms in Finland in 2011 [31]. Raw cow milk producers in Finland are required to test their milk for Listeria monocytogenes, Campylobacter and STEC O26, 0103, O111, 0145 and $\mathrm{O}_{157}$ but not for YP [17]. In this outbreak, the producer's raw milk fulfilled the requirements of the law. Five persons who had consumed this producer's raw milk had fallen ill with campylobacteriosis in March 2014, before the YP outbreak notification. At that time, their illness could not be associated with the raw milk because Campylobacter had not been found in the producer's raw milk. The current testing regime for Campylobacter may be insufficient, because the concentration of the pathogen may be below the threshold of what is detectable in milk. In our investigations in April 2014, an identical C. jejuni genotype was detected in samples of one YP patient, bovine faeces and the producer's milk filter. Examination of milk filters instead of bulk tank milk samples seems to increase the probability of detection of pathogens. In the filter, the concentration of pathogens is higher than in bulk tank milk because large volumes of raw milk flow through it. The milk filter could therefore be used as the standard for testing.

Current control measures for raw milk production are not sufficient to prevent illness in those drinking this product. In this outbreak, contamination occurred although the raw milk met the microbiological criteria. The producer also fulfilled other legal requirements: raw milk was labelled with information on the possible microbial health hazards, risk groups and appropriate storage conditions in order to protect the consumer [17]. Before starting production, the producer is required to prove that the dairy cattle are free from Salmonella and STEC 0157. Specific requirements are in place for the farm buildings and operations on the farm to avoid contamination of milk through contact with manure. The milk has to be cooled immediately after milking and stored at $6^{\circ} \mathrm{C}$ or cooler [17]. The producer also met these requirements. It is relevant to review the legislation and to increase the awareness of the general public and policymakers concerning the potential hazards of raw milk consumption.

\section{Acknowledgements}

We would like to thank all the experts from THL, Evira and the municipal authority who contributed to the outbreak 
investigation and all the questionnaire respondents for taking part in the study. Comments on the manuscript by Noora Tolin and Chris Williams are much appreciated.

\section{Conflict of interest}

None declared.

\section{Authors' contributions}

TP contributed to the collection and analysis of data and drafted the manuscript as the lead writer. $\mathrm{SHa}$ and $\mathrm{MH}$ were responsible for laboratory analysis of food and environmental samples and contributed to the comparison of human, food and environmental samples. SS was responsible for the microbiological investigation of the human samples and contributed to the comparison of human, food and environmental samples. AP contributed to the trace-back investigation. SHe was in charge of the local outbreak investigation. HTN contributed to the local outbreak investigation and was in charge of patient management. JO contributed to the casecontrol study design and statistical analysis of data. SHu contributed to the questionnaire design and data collection. RRF was responsible for the epidemiological investigations and contributed to the case-control study design, questionnaire design and analysis of the data. SS, SHa, AP, MH, SHe, JO and RRF contributed to manuscript writing. All co-authors critically reviewed the draft of the paper and approved the final version.

\section{References}

1. GalindoCL, RosenzweigJA, KirtleyML, ChopraAK. Pathogenesis of $Y$. enterocolitica and Y. pseudotuberculosis in human yersiniosis.J Pathogens. 2011;2011:182051. DOI: 10.4061/2011/182051 PMID: 22567322

2. Heymann DL. Control of communicable diseases manual. 19th ed. Washington: American Public Health Association; 2008.

3. Rimhanen-FinneR, NiskanenT, HallanvuoS, MakaryP, HaukkaK, PajunenS, et al. Yersinia pseudotuberculosis causing a large outbreak associated with carrots in Finland, 2006. Epidemiol Infect. 2009;137(3):342-7. DOI: 10.1017/So950268807000155 PMID: 18177523

4. National Institute for Health and Welfare (THL). Infectious diseases in Finland 2013. Helsinki: THL; 2014. Available from: https://www.julkari.fi/bitstream/handle/10024/125566/URN ISBN_978-952-302-194-5.pdf?sequence=1

5. NuortiJP, NiskanenT, HallanvuoS, Mikkolal, KelaE, HatakkaM, et al. A widespread outbreak of Yersinia pseudotuberculosis 0:3 infection from iceberg lettuce. J Infect Dis. 2004;189(5):766-74. DOI: $10.1086 / 381766$ PMID: 14976592

6. InoueM, NakashimaH, UebaO, IshidaT, DateH, KobashiS, et al. Community outbreak of Yersinia pseudotuberculosis. Microbiol Immunol. 1984;28(8):883-91. DOI: 10.1111/j.1348-0421.1984. tbo0744.x PMID: 6503742

7. David HW, Stanley L. Applied Logistic Regression. 2nd ed. New York: John Wiley and Sons, Inc; 2000.

8. AntikainenJ, KanteleA, PakkanenSH, LääveriT, RiuttaJ, VaaraM, et al. A quantitative polymerase chain reaction assay for rapid detection of 9 pathogens directly from stools of travelers with diarrhea. Clin Gastroenterol Hepatol. 2013;11(10):1300-1307.e3. DOI: 10.1016/j.cgh.2013.03.037 PMID: 23639597

9. LambertzST, NilssonC, HallanvuoS, LindbladM. Real-time PCR method for detection of pathogenic Yersinia enterocolitica in food.Appl Environ Microbiol. 2008;74(19):6060-7. DOI: 10.1128/ AEM.00405-08 PMID: 18708521

10. LambertzST, NilssonC, HallanvuoS. TaqMan-based real-time PCR method for detection of Yersinia pseudotuberculosis in food.Appl Environ Microbiol. 2008;74(20):6465-9. DOI: 10.1128/AEM.01459-08 PMID: 18757572

11. International Organization for Standardization (ISO). ISO/ TS 13136:2012. Microbiology of food and animal feed - Realtime polymerase chain reaction (PCR)-based method for the detection of food-borne pathogens - Horizontal method for the detection of Shiga toxin-producing Escherichia coli (STEC) and the determination of $\mathrm{O}_{157}, \mathrm{O}_{111}, \mathrm{O}_{26}, \mathrm{O}_{103}$ and $\mathrm{O}_{145}$ serogroups. Geneva: ISO; 2012.
12. International Organization for Standardization (ISO). ISO/ WD 10237:2014. Microbiology of the food chain -- Horizontal method for the detection of pathogenic Yersinia enterocolitica. Geneva: ISO; 2014.

13. Weagant SD, Feng P. Bacteriological Analytical Manual: Yersinia enterocolitica. Silver Spring: US Food and Drug Administration; 2007. Available from: http://www.fda.gov/ Food/FoodScienceResearch/LaboratoryMethods/ucm 072633. htm

14. International Organization for Standardization (ISO). ISO 10272-1:2006. Microbiology of food and animal feeding stuffs - Horizontal method for detection and enumeration of Campylobacter spp. - Part 1: Detection method. Geneva: ISO; 2006.

15. HalkilahtiJ, HaukkaK, SiitonenA. Genotyping of outbreakassociated and sporadic Yersinia pseudotuberculosis strains by novel multilocus variable-number tandem repeat analysis (MLVA).J Microbiol Methods. 2013;95(2):245-50. DOI: 10.1016/j. mimet.2013.09.007 PMID: 24050949

16. Centers for Disease Control and Prevention (CDC). Standard operating procedure for PulseNet PFGE of Campylobacter jejuni. Atlanta: CDC; 2013. Available from: http://www.cdc.gov/ pulsenet/PDF/campylobacter-pfge-protocol-508c.pdf

17. Public law. Maa- ja metsätalousministeriön asetus raakamaidon tuotannon ja luovutuksen elintarvikehygieniasta. [Decree of the Ministry of Agriculture and Forestry on food hygiene in the production and supply of raw milk]. Pub. L. No. 699-2013; 26 Sept 2013. Finnish. Available from: http://www. finlex.fi/fi/laki/alkup/2013/20130699\#Pidm1839328

18. Gregg M, Dicker R, Goodman R. Field Epidemiology. 1st ed. New York: Oxford University Press; 1996.

19. NewkirkR, HedbergC, Bender). Establishing a milkborne disease outbreak profile: potential food defense implications. Foodborne Pathog Dis. 2011;8(3):433-7. DOI: 10.1089/ fpd.2010.0731 PMID: 21114422

20. OliverSP, JayaraoBM, AlmeidaRA. Foodborne pathogens in milk and the dairy farm environment: food safety and public health implications. Foodborne Pathog Dis. 2005;2(2):115-29. DOI: 10.1089/fpd.2005.2.115 PMID: 15992306

21. Dennyl, BhatM, EckmannK. Outbreak of Escherichia coli 0157:H7 associated with raw milk consumption in the Pacific Northwest.Foodborne Pathog Dis. 2008;5(3):321-8. DOI: 10.1089/fpd.2007.0072 PMID: 18564912

22. Perkiomäki J, Leimi A, Tuominen P. Suomessa tuotetun raakamaidon biologiset vaarat - riskiprofiili. [Biological hazards of raw milk produced in Finland - risk profile]. Helsinki: Finnish Food Safety Authority Evira; 2012. Finnish. Available from: http://www.evira.fi/portal/fi/tietoa+evirasta/ julkaisut $/ \mathrm{a}=$ view \& productld $=317$

23. Hoogenboom-VerdegaalAM, de JongJC, DuringM, HoogenveenR, HoekstraJA. Community-based study of the incidence of gastrointestinal diseases in The Netherlands. Epidemiol Infect. 1994;112(3):481-7. DOI: 10.1017 / So950268800051189 PMID: 8005214

24. Centers for Disease Control and Prevention (CDC). Raw (unpasteurized) milk. Atlanta: CDC; 2014. Available from: http://www.cdc.gov/features/rawmilk/

25. JalavaK, HakkinenM, ValkonenM, NakariUM, PaloT, HallanvuoS, et al. An outbreak of gastrointestinal illness and erythema nodosum from grated carrots contaminated with Yersinia pseudotuberculosis. J Infect Dis. 2006;194(9):1209-16. DOI: 10.1086/508191 PMID: 17041846

26. Felming D, Hunt D. Biological safety principles and practices. 4th ed. Washington: ASM Press; 2006.

27. Bottone EJ, Bercovier H, Mollaret HH. Bergey's Manual of Systematic Bacteriology. 2nd ed. New York: Springer; 2005.

28. European Food Safety Authority (EFSA). Scientific opinion on the public health risks related to the consumption of raw drinking milk. Parma: EFSA; 2015. Available from: http://www. efsa.europa.eu/en/efsajournal/doc/3940.pdf

29. McAuleyCM, McMillanK, MooreSC, FeganN, FoxEM. Prevalence and characterization of foodborne pathogens from Australian dairy farm environments.J Dairy Sci. 2014;97(12):7402-12. DOI: 10.3168/jds.2014-8735 PMID: 25282417

30. QuigleyL, McCarthyR, O'SullivanO, BeresfordTP, FitzgeraldGF, RossRP, et al. The microbial content of raw and pasteurized cow milk as determined by molecular approaches. J Dairy Sci. 2013;96(8):4928-37. DOI: 10.3168/jds.2013-6688 PMID: 23746589

31. RuusunenM, SalonenM, PulkkinenH, HuuskonenM, HellströmS RevezJ, et al. Pathogenic bacteria in Finnish bulk tank milk. Foodborne Pathog Dis. 2013;10(2):99-106. DOI: 10.1089/ fpd.2012.1284 PMID: 23373473 